\title{
Generalised polygons admitting a point-primitive almost simple group of Suzuki or Ree type
}

\author{
Luke Morgan* Tomasz Popiel ${ }^{\dagger}$ \\ Centre for the Mathematics of Symmetry and Computation \\ School of Mathematics and Statistics \\ The University of Western Australia \\ 35 Stirling Highway, Crawley, WA 6009 Australia \\ \{luke.morgan, tomasz.popiel\}@uwa.edu.au
}

Submitted: Aug 24, 2015; Accepted: Feb 8, 2016; Published: Feb 19, 2016

Mathematics Subject Classifications: 51E12, 20B15, 05B25

\begin{abstract}
Let $G$ be a collineation group of a thick finite generalised hexagon or generalised octagon $\Gamma$. If $G$ acts primitively on the points of $\Gamma$, then a recent result of Bamberg et al. shows that $G$ must be an almost simple group of Lie type. We show that, furthermore, the minimal normal subgroup $S$ of $G$ cannot be a Suzuki group or a Ree group of type ${ }^{2} \mathrm{G}_{2}$, and that if $S$ is a Ree group of type ${ }^{2} \mathrm{~F}_{4}$, then $\Gamma$ is (up to point-line duality) the classical Ree-Tits generalised octagon.
\end{abstract}

Keywords: generalised hexagon; generalised octagon; primitive permutation group

\section{Introduction}

A generalised $d$-gon is a point-line incidence geometry $\Gamma$ whose bipartite incidence graph has diameter $d$ and girth $2 d$. If each point of $\Gamma$ is incident with at least three lines, and each line is incident with at least three points, then $\Gamma$ is said to be thick. By the well-known Feit-Higman Theorem [6], thick finite generalised $d$-gons exist only for $d \in\{2,3,4,6,8\}$. In the present paper, we are concerned with the cases $d=6$ (generalised hexagons), and $d=8$ (generalised octagons).

A collineation (or automorphism) of $\Gamma$ is a permutation of the point set of $\Gamma$, together with a permutation of the line set, such that the incidence relation is preserved (equivalently, an automorphism of the incidence graph of $\Gamma$ that preserves the parts). The only known thick finite generalised hexagons and octagons arise as natural geometries

\footnotetext{
*Supported by ARC grant DP120100446.
}

${ }^{\dagger}$ Supported by ARC grant DP140100416. 
for certain exceptional groups of Lie type: $\mathrm{G}_{2}(q)$ and ${ }^{3} \mathrm{D}_{4}(q)$ are collineation groups of generalised hexagons, and ${ }^{2} \mathrm{~F}_{4}(q)$ acts on a generalised octagon. In each case, the action of the collineation group is primitive on both the points and the lines of $\Gamma$, and transitive on the flags of $\Gamma$, namely the incident point-line pairs. Each action is also point-distance-transitive - that is, transitive on each set of ordered pairs of points at a given distance from each other in the incidence graph - and line-distance-transitive. Buekenhout and Van Maldeghem [4] showed that point-distance-transitivity implies pointprimitivity for a thick finite generalised hexagon or octagon, and proved that there exist no point-distance-transitive examples other than the known classical examples. The existence of other point-primitive or flag-transitive (thick finite) generalised hexagons or octagons remains an open question.

Schneider and Van Maldeghem [10] showed that a group $G$ acting point-primitively, line-primitively, and flag-transitively on a thick finite generalised hexagon or octagon must be an almost simple group of Lie type. That is, $S \leqslant G \leqslant \operatorname{Aut}(S)$, with $S$ a finite simple group of Lie type. Bamberg et al. [1] then showed that point-primitivity alone is sufficient to imply the same conclusion. We continue this work here, treating the families of Lie type groups that are of fixed rank and fixed characteristic.

Theorem 1. Let $G$ be a point-primitive collineation group of a thick finite generalised hexagon or generalised octagon $\Gamma$, with $S \leqslant G \leqslant \operatorname{Aut}(S)$ for some nonabelian finite simple group $S$. Then $S$ is not a Suzuki group or a Ree group of type ${ }^{2} \mathrm{G}_{2}$. Moreover, if $S$ is a Ree group of type ${ }^{2} \mathrm{~F}_{4}$, then, up to point-line duality, $\Gamma$ is isomorphic to the classical Ree-Tits generalised octagon.

The non-simple groups ${ }^{2} \mathrm{~B}_{2}(2),{ }^{2} \mathrm{G}_{2}$ or ${ }^{2} \mathrm{~F}_{4}(2)$ are not treated by the above theorem. We refer the reader to [3], where generalised hexagons and generalised octagons admitting almost simple groups with socle ${ }^{2} \mathrm{G}_{2}(2)^{\prime}$ and ${ }^{2} \mathrm{~F}_{4}(2)^{\prime}$ were investigated.

Theorem 1 is proved in three sections: the Suzuki groups are considered in Section 3; the small and large Ree groups are dealt with in Sections 4 and 5, respectively.

\section{Preliminaries}

Let us first collect some basic facts and definitions. If a finite generalised hexagon or octagon $\Gamma$ is thick, then there exist constants $s, t \geqslant 2$ such that each point (line) of $\Gamma$ is incident with exactly $t+1$ lines $(s+1$ points), and $(s, t)$ is called the order of $\Gamma$. If $\mathcal{P}$ denotes the point set of $\Gamma$, then $[11$, p. 20]

$$
|\mathcal{P}|= \begin{cases}(s+1)\left(s^{2} t^{2}+s t+1\right) & \text { if } \Gamma \text { is a generalised hexagon, } \\ (s+1)(s t+1)\left(s^{2} t^{2}+1\right) & \text { if } \Gamma \text { is a generalised octagon. }\end{cases}
$$

Moreover, the integers st and $2 s t$ are squares in the respective cases where $\Gamma$ is a generalised hexagon or generalised octagon.

Lemma 2. Let $\mathcal{P}$ be the point set of a thick finite generalised hexagon or generalised octagon $\Gamma$. 
(i) If $2^{a}$ divides $|\mathcal{P}|$, where $a \geqslant 1$, then $|\mathcal{P}|>2^{3 a}$.

(ii) If $\Gamma$ is a generalised hexagon and $3^{a}$ divides $|\mathcal{P}|$, where $a \geqslant 1$, then $|\mathcal{P}|>3^{3 a-4}$.

(iii) If $\Gamma$ is a generalised octagon and $2^{a} 3^{b}$ divides $|\mathcal{P}|$, where $a \geqslant 0$ and $b \geqslant 1$, then $|\mathcal{P}|>2^{a} 3^{2 b}$.

Proof. Let $(s, t)$ be the order of $\Gamma$.

(i) First suppose that $\Gamma$ is a generalised hexagon. Since $s^{2} t^{2}+s t+1$ is odd, $2^{a}$ must divide $s+1$. In particular, $s+1 \geqslant 2^{a}$, and hence $s \geqslant 2^{a-1}$. Therefore, $|\mathcal{P}|>$ $(s+1) s^{2} t^{2} \geqslant 2^{a}\left(2^{a-1}\right)^{2} 2^{2}=2^{3 a}$. Now let $\Gamma$ be a generalised octagon. Since $2 s t$ is a square, st must be even, so $(s t+1)\left(s^{2} t^{2}+1\right)$ is odd, and hence $2^{a}$ must divide $s+1$. Therefore, $|\mathcal{P}|>(s+1) s^{3} t^{3} \geqslant 2^{a}\left(2^{a-1}\right)^{3} 2^{3}=2^{4 a}>2^{3 a}$.

(ii) Since $s^{2} t^{2}+s t+1$ is not divisible by $9, s+1$ must be divisible by $3^{a-1}$. In particular, $s+1 \geqslant 3^{a-1}$, and hence $s \geqslant 3^{a-2}$. Therefore, $|\mathcal{P}|>(s+1) s^{2} t^{2} \geqslant 3^{a-1}\left(3^{a-2}\right)^{2} 2^{2}>3^{3 a-4}$.

(iii) Since $2 s t$ is a square, st is even, so $s^{2} t^{2}+1$ is divisible by neither 2 nor 3 . Hence, $2^{a} 3^{b}$ divides $(s+1)(s t+1)$. In particular, $(s+1)(s t+1) \geqslant 2^{a} 3^{b}$. Let us say that $s+1$ is divisible by $3^{c}$, and that $s t+1$ is divisible by $3^{d}$, where $c+d=b$. If $c \geqslant 1$, then $s>3^{c-1 / 2}$; and if $d \geqslant 1$, then $s t>3^{d-1 / 2}$. Also, $t-1=(s t+1)-(s+1) t$ is divisible by $3^{\min \{c, d\}}$, so $t>3^{\min \{c, d\}}$. If $c \geqslant d$, then $c \geqslant 1$ and $t>3^{d}$, and hence $|\mathcal{P}|>(s+1)(s t+1)(s t)^{2} \geqslant 2^{a} 3^{b}\left(3^{c-1 / 2} 3^{d}\right)^{2}=2^{a} 3^{b+2(c+d)-1}=2^{a} 3^{3 b-1} \geqslant 2^{a} 3^{2 b}$. If $d>c$, then $d \geqslant(b+1) / 2$, so $|\mathcal{P}|>(s+1)(s t+1)(s t)^{2} \geqslant 2^{a} 3^{b}\left(3^{d-1 / 2}\right)^{2} \geqslant 2^{a} 3^{b}\left(3^{b / 2}\right)^{2}=2^{a} 3^{2 b}$.

Recall that a permutation group $G \leqslant \operatorname{Sym}(\Omega)$ acts primitively on the set $\Omega$ if it acts transitively and preserves no nontrivial partition of $\Omega$, and that this is equivalent to the stabiliser $G_{\omega}$ of a point $\omega \in \Omega$ being a maximal subgroup of $G$. A maximal subgroup $M$ of an almost simple group $G$ with minimal normal subgroup $S$ is said to be a novelty maximal subgroup if $S \cap M$ is not maximal in $S$. Our notation is mostly standard: we write $D_{n}$ for a dihedral group of order $n ; C_{n}$ denotes a cyclic group of order $n ;[n]$ denotes an unspecified group of order $n$; and, for $q$ a prime power, $E_{q}$ denotes an elementary abelian group of order $q$. For information about the Suzuki and Ree simple groups of Lie type, we refer the reader to [13], and the other references mentioned below.

\section{Proof of Theorem 1: $S$ a Suzuki group}

We now adopt the hypothesis of Theorem 1, assuming additionally that $S$ is isomorphic to $\mathrm{Sz}(q)={ }^{2} \mathrm{~B}_{2}(q)$, where $q=2^{m}$ with $m$ odd and at least 3 . (We exclude the case $m=1$ because ${ }^{2} \mathrm{~B}_{2}(2)$ is soluble.) Then

$$
|S|=q^{2}\left(q^{2}+1\right)(q-1)=q^{2}(q+\sqrt{2 q}+1)(q-\sqrt{2 q}+1)(q-1) .
$$

The outer automorphism group of $S$ is cyclic of order $m$. If we let $\sigma$ denote a generator of this group, then we have $G=S:\left\langle\sigma^{j}\right\rangle$ for some divisor $j$ of $m$. Let $\mathcal{P}$ be the point set of $\Gamma$, and let $x \in \mathcal{P}$. Observe first that the stabiliser $G_{x}$ cannot contain $S$ : if it did, then $G_{x}$ would have the form $S: K$ for some maximal subgroup $K$ of the cyclic group $\left\langle\sigma^{j}\right\rangle$, and 
hence $\left|G: G_{x}\right|=|\mathcal{P}|$ would be a prime, which is seen to be impossible upon inspection of (1). Now, as explained in [2, Section 7.3], $G$ has no novelty maximal subgroups. Therefore, $S_{x}=G_{x} \cap S$ is a maximal subgroup of $S$, so $S$ itself acts primitively on $\mathcal{P}$, and hence to prove the theorem we may assume that $G=S$. The maximal subgroups of $S$ are $[2$, Table 8.16], up to conjugacy,

(i) $E_{q} \cdot E_{q} \cdot C_{q-1}$,

(ii) $D_{2(q-1)}$,

(ii) $C_{q \pm \sqrt{2 q}+1}: C_{4}$

(iv) $\operatorname{Sz}\left(q_{0}\right)$, where $q=q_{0}^{r}$ with $r$ prime and $q_{0}>2$.

\subsection{Case (i)}

Suppose that $S_{x} \cong E_{q} \cdot E_{q} \cdot C_{q-1}$. Suzuki [12] showed that $S$ is 2 -transitive in this action. Since $S$ preserves the incidence relation on $\Gamma$, and therefore distance in the incidence graph of $\Gamma$, we have that the diameter of the incidence graph is at most three, a contradiction.

\subsection{Cases (ii) $-(\mathrm{iv})$}

For the remaining cases, we apply Lemma 2(i). If $S_{x} \cong D_{2(q-1)}$, then

$$
|\mathcal{P}|=\left|S: S_{x}\right|=\frac{1}{2} q^{2}\left(q^{2}+1\right)=2^{2 m-1}\left(2^{2 m}+1\right)<2^{4 m},
$$

contradicting Lemma $2(\mathrm{i})$ with $a=2 m-1$, which says that $|\mathcal{P}|>2^{6 m-3}$.

If $S_{x} \cong C_{q \pm \sqrt{2 q}+1}: C_{4}$, then

$$
|\mathcal{P}|=\left|S: S_{x}\right|=\frac{1}{4} q^{2}(q \mp \sqrt{2 q}+1)(q-1)=2^{2 m-2}\left(2^{m} \mp 2^{(m+1) / 2}+1\right)\left(2^{m}-1\right)<2^{4 m-1},
$$

contradicting Lemma $2(\mathrm{i})$ with $a=2 m-2$, which says that $|\mathcal{P}|>2^{6 m-6}$.

Finally, suppose that $S_{x} \cong \operatorname{Sz}\left(q_{0}\right)$, where $q=q_{0}^{r}$ with $r$ prime and $q_{0}>2$. Writing $q_{0}=2^{\ell}$, we have

$$
|\mathcal{P}|=\left|S: S_{x}\right|=2^{2 \ell(r-1)} \frac{\left(2^{2 \ell r}+1\right)\left(2^{\ell r}-1\right)}{\left(2^{2 \ell}+1\right)\left(2^{\ell}-1\right)}<2^{5 \ell(r-1)+2}
$$

contradicting Lemma $2(\mathrm{i})$ with $a=2 \ell(r-1)$, which says that $|\mathcal{P}|>2^{6 \ell(r-1)}$.

\section{Proof of Theorem 1: $S$ a Ree group of type ${ }^{2} \mathrm{G}_{2}$}

We now adopt the hypothesis of Theorem 1 and assume that $S \cong{ }^{2} \mathrm{G}_{2}(q)$, where $q=3^{m}$ with $m$ odd and at least 3 . (We exclude the case $m=1$ because ${ }^{2} \mathrm{G}_{2}(3)$ is not simple.) Then

$$
|S|=q^{3}\left(q^{3}+1\right)(q-1)=q^{3}(q+\sqrt{3 q}+1)(q-\sqrt{3 q}+1)\left(q^{2}-1\right) .
$$


Let $\mathcal{P}$ be the point set of $\Gamma$, and let $x \in \mathcal{P}$. The outer automorphism group of $S$ is cyclic (of order $m$ ), so, as in Section 3, we first deduce that $G_{x}$ is a maximal subgroup of $G$ not containing $S$. The maximal subgroups of $G$ were determined by Kleidman [8, Theorem C]. In particular, $G$ has no novelty maximal subgroups, so it suffices to prove the theorem in the case where $G=S$. The maximal subgroups of $S$ are, up to conjugacy,

(i) $E_{q} \cdot E_{q} \cdot E_{q} \cdot C_{q-1}$,

(ii) $C_{2} \times \operatorname{PSL}_{2}(q)$,

(iii) $\left(E_{4} \times D_{(q+1) / 2}\right): C_{3}$,

(iv) $C_{q \pm \sqrt{3 q}+1}: C_{6}$

(v) ${ }^{2} \mathrm{G}_{2}\left(q_{0}\right)$, where $q=q_{0}^{r}$ with $r$ prime.

\subsection{Case (i)}

Suppose that $S_{x} \cong E_{q} \cdot E_{q} \cdot E_{q} \cdot C_{q-1}$. Then $S$ acts 2 -transitively on $\mathcal{P}$ [5, p. 251]. The same argument as in Section 3.1 now provides a contradiction.

\section{2 $\Gamma$ a generalised hexagon: cases (ii) $-(\mathrm{v})$}

For cases (ii)-(v) with $\Gamma$ a generalised hexagon, we use Lemma 2(ii). First suppose that $S_{x} \cong C_{2} \times \operatorname{PSL}_{2}(q)$. The order of $S_{x}$ is $q\left(q^{2}-1\right)$, so

$$
|\mathcal{P}|=\left|S: S_{x}\right|=q^{2}\left(q^{2}-q+1\right)=3^{2 m}\left(3^{2 m}-3^{m}+1\right)<3^{4 m},
$$

contradicting Lemma 2(ii) with $a=2 m$, which says that $|\mathcal{P}|>3^{6 m-4}$.

If $S_{x} \cong\left(E_{4} \times D_{(q+1) / 2}\right): C_{3}$, then

$$
|\mathcal{P}|=\left|S: S_{x}\right|=\frac{1}{6} q^{3}(q-1)\left(q^{2}-q+1\right)=\frac{1}{2} 3^{3 m-1}\left(3^{m}-1\right)\left(3^{2 m}-3^{m}+1\right)<3^{6 m-1},
$$

contradicting Lemma 2(ii) with $a=3 m-1$, which says that $|\mathcal{P}|>3^{9 m-7}$.

If $S_{x} \cong C_{q \pm \sqrt{3 q}+1}: C_{6}$, then

$$
|\mathcal{P}|=\left|S: S_{x}\right|=q^{3}\left(q^{2}-1\right)(q \mp \sqrt{3 q}+1)=3^{3 m}\left(3^{2 m}-1\right)\left(3^{m} \mp 3^{(m+1) / 2}+1\right)<3^{6 m+1},
$$

contradicting Lemma 2(ii) with $a=3 m$, which says that $|\mathcal{P}|>3^{9 m-4}$.

Finally, suppose that $S_{x} \cong{ }^{2} G_{2}\left(q_{0}\right)$, where $q=q_{0}^{r}$ with $r$ prime. Writing $q_{0}=3^{\ell}$, we have

$$
|\mathcal{P}|=\left|S: S_{x}\right|=3^{3 \ell(r-1)} \frac{\left(3^{3 \ell r}+1\right)\left(3^{\ell r}-1\right)}{\left(3^{3 \ell}+1\right)\left(3^{\ell}-1\right)}<3^{7 \ell(r-1)+2} .
$$

If $\ell(r-1) \geqslant 3$, then this contradicts Lemma 2(ii) with $a=3 \ell(r-1)$, which gives $|\mathcal{P}|>$ $3^{9 \ell(r-1)-4}$. Otherwise, $(\ell, r)=(1,3)$, and there is no valid solution $(s, t)$ to equation $(1)$. 


\section{3 $\Gamma$ a generalised octagon: cases (ii) - (iv)}

Now suppose that $\Gamma$ is a generalised octagon. We first use Lemma 2(iii) to rule out cases (ii)-(iv) for $S_{x}$, computing $\left|S: S_{x}\right|$ in each case as in Section 4.2. First suppose that $S_{x} \cong C_{2} \times \mathrm{PSL}_{2}(q)$. Then

$$
|\mathcal{P}|=\left|S: S_{x}\right|=3^{2 m}\left(3^{2 m}-3^{m}+1\right)<3^{4 m},
$$

contradicting Lemma 2(iii) with $a=0$ and $b=2 m$, which says that $|\mathcal{P}|>3^{4 m}$.

Next, suppose that $S_{x} \cong\left(E_{4} \times D_{(q+1) / 2}\right): C_{3}$. Observe that $3^{3 m}+1$ is divisible by 4 , because $3 m$ is odd. Therefore,

$$
|\mathcal{P}|=\left|S: S_{x}\right|=2 \cdot 3^{3 m-1} \frac{3^{3 m}+1}{4}<2 \cdot 3^{6 m-2},
$$

while Lemma 2(iii) with $a=1$ and $b=3 m-1$ gives $|\mathcal{P}|>2 \cdot 3^{6 m-2}$, a contradiction.

Finally, suppose that $S_{x} \cong C_{q \pm \sqrt{3 q}+1}: C_{6}$. Observe that $3^{2 m}-1$ is divisible by $2^{3}$ because $m$ is odd, and that $3^{m} \mp 3^{(m+1) / 2}+1$ is even. Therefore,

$$
\begin{aligned}
|\mathcal{P}|=\left|S: S_{x}\right| & =2^{4} 3^{3 m} \frac{\left(3^{2 m}-1\right)\left(3^{m} \mp 3^{(m+1) / 2}+1\right)}{2^{4}} \\
& \leqslant 2^{4} 3^{3 m} \frac{\left(3^{2 m}-1\right)\left(3^{m}+3^{(m+1) / 2}+1\right)}{2^{4}}<2^{4} 3^{6 m-2},
\end{aligned}
$$

while Lemma 2(iii) with $a=4$ and $b=3 m$ gives $|\mathcal{P}|>2^{4} 3^{6 m}$, a contradiction.

\section{4 $\Gamma$ a generalised octagon: case $(\mathrm{v})$}

Finally, we consider case (v) with $\Gamma$ a generalised octagon. The approach is similar to that used for cases (ii)-(iv), but requires a little more care.

Suppose that $S_{x} \cong{ }^{2} \mathrm{G}_{2}\left(q_{0}\right)$, where $q=q_{0}^{r}$ with $r$ prime. Writing $q_{0}=3^{\ell}$, we have

$$
|\mathcal{P}|=3^{3 \ell(r-1)} \frac{\left(3^{3 \ell r}+1\right)\left(3^{\ell r}-1\right)}{\left(3^{3 \ell}+1\right)\left(3^{\ell}-1\right)}<3^{7 \ell(r-1)+\epsilon}, \quad \text { where } \epsilon:=\frac{\log \left(\frac{3^{4}}{\left(3^{3}-1\right)(3-1)}\right)}{\log (3)} \approx 0.336
$$

To verify the inequality in $(2)$, one checks that $\left(3^{3 \ell}+1\right)\left(3^{\ell}-1\right) \geqslant 3^{4 \ell-\epsilon}$, because $\ell \geqslant 1$, and that $\left(3^{3 \ell r}+1\right)\left(3^{\ell r}-1\right)<3^{4 \ell r}$. Let us re-write this inequality as

$$
|\mathcal{P}|<3^{7 b / 3+\epsilon}, \quad \text { where } b:=3 \ell(r-1) .
$$

Note also that $b \geqslant 6$, because $r \geqslant 3$. For a contradiction, we now show that $|\mathcal{P}|>3^{7 b / 3+\epsilon}$. By $(2), 3^{b}$ is the highest power of 3 dividing $|\mathcal{P}|$. Since $2 s t$ is a square, st is even, so $s^{2} t^{2}+1$ is not divisible by 3 . Hence, by $(1), 3^{b}$ divides $(s+1)(s t+1)$. As in the proof of Lemma 2(iii), let us say that $s+1$ is divisible by $3^{c}$, and that $s t+1$ is divisible by $3^{d}$, where $c+d=b$. Recall also (from that proof) that $t>3^{\min \{c, d\}}$. To show that $|\mathcal{P}|>3^{7 b / 3+\epsilon}$, we consider four cases. 
First suppose that $c \geqslant d$. Then $t>3^{d}$, and $c \geqslant 1$ so $s \geqslant 3^{c}-1>3^{c-1 / 2}$. Hence, $|\mathcal{P}|>(s+1)(s t+1)(s t)^{2}>3^{b}\left(3^{c-1 / 2} 3^{d}\right)^{2}=3^{b+2(c+d)-1}=3^{3 b-1}>3^{7 b / 3+1}$, with the final inequality holding because $b \geqslant 6>3$. Next, suppose that $d / 2+1 / 2 \leqslant c<d$. Then $6 \leqslant b=c+d \leqslant 3 c-1$. In particular, $c \geqslant(b+1) / 3$; and $c \geqslant 3$ so $s \geqslant 3^{c}-1 \geqslant 3^{c-\delta}$, where $\delta:=3-\log \left(3^{3}-1\right) / \log (3)$. Moreover, $t>3^{c}$, and hence $|\mathcal{P}|>3^{b}(s t)^{2}>3^{b}\left(3^{c-\delta} 3^{c}\right)^{2}=$ $3^{b+4 c-2 \delta} \geqslant 3^{7 b / 3+(4 / 3-2 \delta)}$. It follows that $|\mathcal{P}|>3^{7 b / 3+\epsilon}$, because $1.26 \approx 4 / 3-2 \delta>\epsilon \approx 0.336$. Now suppose that $c \leqslant d / 2-1 / 2$. Then $6 \leqslant b=c+d \leqslant 3 d / 2-1 / 2$. In particular, $d \geqslant(2 b+1) / 3$; and $d \geqslant 5$ so $s t \geqslant 3^{d}-1 \geqslant 3^{d-\delta^{\prime}}$, where $\delta^{\prime}:=5-\log \left(3^{5}-1\right) / \log (3)$. Therefore, $|\mathcal{P}|>3^{b}(s t)^{2}>3^{b+2 d-2 \delta^{\prime}}=3^{7 b / 3+2 / 3-2 \delta^{\prime}}$, and it follows that $|\mathcal{P}|>3^{7 b / 3+\epsilon}$, because $0.659 \approx 2 / 3-2 \delta^{\prime}>\epsilon \approx 0.336$.

Finally, suppose that $d / 2-1 / 2<c<d / 2+1 / 2$. Since $c$ and $d$ are integers, this is equivalent to saying that $c=d / 2$. Now, suppose first, towards a contradiction, that $(s+1)(s t+1)$ is actually equal to $3^{b}$. Then $s+1=3^{c}$, st $+1=3^{2 c}$, and (2) implies that

$$
\left(s^{2} t^{2}+1\right)\left(3^{3 \ell}+1\right)\left(3^{\ell}-1\right)=\left(3^{3 \ell r}+1\right)\left(3^{\ell r}-1\right) .
$$

However, this is impossible, because the left- and right-hand sides of (3) are not congruent modulo 3. Indeed, $s t=3^{2 c}-1 \equiv 2(\bmod 3)$, so $s^{2} t^{2}+1 \equiv 4+1 \equiv 2(\bmod 3) ; 3^{3 \ell}+1 \equiv 1$ $(\bmod 3)$; and $3^{\ell}-1 \equiv 2(\bmod 3)$; and hence the left-hand side of $(3)$ is congruent to 1 modulo 3 . On the other hand, the right-hand side of (3) is congruent to 2 modulo 3 . Therefore, $(s+1)(s t+1)$ is strictly larger than $3^{b}$. Indeed, it is larger by a factor of at least 5 , because by $(2)$ we see that $|\mathcal{P}| / 3^{b}$ is divisible by neither 2 nor 3 . (To verify that $|\mathcal{P}| / 3^{b}$ is odd, apply [7, Lemma 2.5].) Therefore, $|\mathcal{P}|>5 \cdot 3^{b}(s t)^{2}>3^{b+1}(s t)^{2}$. Since $6 \leqslant b=3 d / 2$, we have $d \geqslant 4$, and so $s t \geqslant 3^{d}-1 \geqslant 3^{d-\delta^{\prime \prime}}$, where $\delta^{\prime \prime}:=4-\log \left(3^{4}-1\right) / \log (3)$.

Hence, $|\mathcal{P}|>3^{b+1+2 d-2 \delta^{\prime \prime}}=3^{7 b / 3+1-2 \delta^{\prime \prime}}$, and it follows that $|\mathcal{P}|>3^{7 b / 3+\epsilon}$, because $0.977 \approx$ $1-2 \delta^{\prime \prime}>\epsilon \approx 0.336$.

\section{Proof of Theorem 1: $S$ a Ree group of type ${ }^{2} \mathrm{~F}_{4}$}

In this final section, we adopt the hypothesis of Theorem 1 while assuming that $S \cong$ ${ }^{2} \mathrm{~F}_{4}(q)$, where $q=2^{m}$ with $m$ odd and at least 3 . (We exclude the case $m=1$ because ${ }^{2} \mathrm{~F}_{4}(2)$ is not simple.) Then

$$
|S|=q^{12}\left(q^{6}+1\right)\left(q^{4}-1\right)\left(q^{3}+1\right)(q-1) .
$$

Let $\mathcal{P}$ be the point set of $\Gamma$, and let $x \in \mathcal{P}$. The outer automorphism group of $S$ is cyclic, so we again observe that $G_{x}$ is a maximal subgroup of $G$ not containing $S$. A result of Malle [9] tells us that $G$ has no novelty maximal subgroups, so it again suffices to prove the theorem in the case where $G=S$. The maximal subgroups of $S$ (listed also in [13, Section 4.9.3]) are, up to conjugacy,

(i) $P_{1}:=\left[q^{10}\right]:\left(\mathrm{Sz}(q) \times C_{q-1}\right)$,

(ii) $P_{2}:=\left[q^{11}\right]: \mathrm{GL}_{2}(q)$, 
(iii) $\mathrm{SU}_{3}(q): C_{2}$,

(iv) $\mathrm{PGU}_{3}(q): C_{2}$,

(v) $\operatorname{Sz}(q) \imath C_{2}$,

(vi) $\operatorname{Sp}_{4}(q): C_{2}$,

(vii) ${ }^{2} \mathrm{~F}_{4}\left(q_{0}\right)$, where $q=q_{0}^{r}$ with $r$ prime,

(viii) $\left(C_{q+1} \times C_{q+1}\right): \mathrm{GL}_{2}(3)$,

(ix) $C_{(q \pm \sqrt{2 q}+1)^{2}}:[96]$,

(x) $C_{q^{2}+q+1 \pm \sqrt{2 q}(q+1)}: C_{12}$.

The groups $P_{1}$ and $P_{2}$ are maximal parabolic subgroups of $S$. The group $P_{1}$ is a point stabiliser in the action of $S$ on the classical generalised octagon, whilst $P_{2}$ is a point stabiliser in the action of $S$ on the dual [13, Section 4.9.4]. We must show that $S_{x}$ cannot be isomorphic to any of the groups in cases (iii)-(x), and, further, that if $S_{x}$ is isomorphic to either $P_{1}$ or $P_{2}$, then $\Gamma$ is the classical generalised octagon or its dual.

\subsection{Cases (i)-(ii) with $\Gamma$ a generalised octagon}

Suppose that $\Gamma$ is a generalised octagon and that $S_{x}$ is isomorphic to either $P_{1}$ or $P_{2}$. In either action, the group $S$ has rank five. That is, the point stabiliser $S_{x}$ has five orbits on the set $\mathcal{P}\left[13\right.$, Section 4.9.4]. For $i \in\{0,2,4,6,8\}$, denote by $\Gamma_{i}(x)$ the set of points at distance $i$ from $x$ in the incidence graph of $\Gamma$. Since each of these sets is nontrivial and $S_{x}$-invariant, the pigeonhole principle shows that each is an orbit of $S_{x}$. Since $S$ acts transitively on $\mathcal{P}$, we find that $S$ acts distance-transitively on $\mathcal{P}$. Now the main result of [4] shows that $\Gamma$ is isomorphic to the classical generalised octagon associated with $S$, or its dual.

\subsection{Case (i) with $\Gamma$ a generalised hexagon}

Suppose that $\Gamma$ is a generalised hexagon, with $S_{x} \cong\left[q^{10}\right]:\left(\operatorname{Sz}(q) \times C_{q-1}\right)$. Since $|\operatorname{Sz}(q)|=$ $q^{2}\left(q^{2}+1\right)(q-1)$

$$
|\mathcal{P}|=\left|S: S_{x}\right|=\left(q^{4}-q^{2}+1\right)\left(q^{3}+1\right)\left(q^{2}+1\right)(q+1) .
$$

Equivalently (subtracting 1 from both sides),

$$
s^{3} t^{2}+s^{2}(t+1)+s(t+1)=q^{10}+q^{9}+q^{7}+q^{6}+q^{4}+q^{3}+q .
$$

Now, $S$ acts primitively and distance-transitively on the points of a generalised octagon of order $\left(q, q^{2}\right)$, with point stabiliser $\left[q^{10}\right]:\left(\mathrm{Sz}(q) \times C_{q-1}\right)$ and nontrivial subdegrees [13, Section 4.9.4]

$$
n_{1}:=q\left(q^{2}+1\right), \quad n_{2}:=q^{4}\left(q^{2}+1\right), \quad n_{3}:=q^{7}\left(q^{2}+1\right), \quad n_{4}:=q^{10} .
$$


Recall the notation $\Gamma_{i}(x)$ from Section 5.1. Then we have [11, p. 19]

$$
\left|\Gamma_{2}(x)\right|=s(t+1), \quad\left|\Gamma_{4}(x)\right|=s^{2} t(t+1), \quad\left|\Gamma_{6}(x)\right|=s^{3} t^{2},
$$

and $S_{x}$ preserves the sets $\Gamma_{i}(x)$. Hence, each $\Gamma_{i}(x)$ is a union of $S_{x}$-orbits, and so for $i \in\{2,4,6\}$, we have $\left|\Gamma_{i}(x)\right|=\sum_{k=1}^{4} \delta_{i, k} n_{k}$, for some $\delta_{i, k} \in\{0,1\}$ (with $\delta_{i, k} \delta_{j, k}=0$ for $i \neq j$ ). We show that this leads to a contradiction.

Claim 1: $\left|\Gamma_{2}(x)\right|=n_{1}$. The proof of the claim is by contradiction. If not, then $\left|\Gamma_{2}(x)\right| \geqslant n_{2}=q^{4}\left(q^{2}+1\right)$. Since $s, t \geqslant 2$, and so in particular $t \geqslant \frac{2}{3}(t+1)$, it follows that

$$
\begin{aligned}
& \left|\Gamma_{4}(x)\right| \geqslant \frac{2}{3} s^{2}(t+1)^{2}=\frac{2}{3}\left|\Gamma_{2}(x)\right|^{2} \geqslant \frac{2}{3} q^{8}\left(q^{2}+1\right)^{2}, \\
& \left|\Gamma_{6}(x)\right| \geqslant 2 s^{2} t^{2} \geqslant \frac{4}{3}\left|\Gamma_{4}(x)\right| \geqslant \frac{8}{9} q^{8}\left(q^{2}+1\right)^{2} .
\end{aligned}
$$

Since the left-hand side of (4) is $\left|\Gamma_{2}(x)\right|+\left|\Gamma_{4}(x)\right|+\left|\Gamma_{6}(x)\right|$, this implies that

$$
\frac{14}{9} q^{8}\left(q^{2}+1\right)^{2}+q^{4}\left(q^{2}+1\right) \leqslant q^{10}+q^{9}+q^{7}+q^{6}+q^{4}+q^{3}+q,
$$

which is certainly false for $q \geqslant 8$.

Claim 2: $\left|\Gamma_{4}(x)\right|=n_{2}$. The proof is again by contradiction. If not, then $\left|\Gamma_{4}(x)\right| \geqslant$ $n_{3}=q^{7}\left(q^{2}+1\right)$, because $\left|\Gamma_{2}(x)\right|=n_{1}=q\left(q^{2}+1\right)$ by Claim 1. This implies the following inequality, which is certainly false for $q \geqslant 8$ :

$$
q^{6}=\frac{q^{7}\left(q^{2}+1\right)}{q\left(q^{2}+1\right)} \leqslant \frac{\left|\Gamma_{4}(x)\right|}{\left|\Gamma_{2}(x)\right|}=\frac{s^{2} t(t+1)}{s(t+1)}=s t<s(t+1)=q\left(q^{2}+1\right) .
$$

By Claims 1 and 2, we must have $\left|\Gamma_{6}(x)\right|=n_{3}+n_{4}=q^{7}\left(q^{3}+q^{2}+1\right)>q^{8}\left(q^{2}+1\right)$, and hence

$$
s>\frac{s^{3} t^{2}}{s^{2} t(t+1)}=\frac{\left|\Gamma_{6}(x)\right|}{\left|\Gamma_{4}(x)\right|}>\frac{q^{8}\left(q^{2}+1\right)}{q^{4}\left(q^{2}+1\right)}=q^{4} .
$$

This is impossible, because $s(t+1)=q\left(q^{2}+1\right)$ by Claim 1 (and hence certainly $s<$ $\left.q\left(q^{2}+1\right)<q^{4}\right)$.

\subsection{Case (ii) with $\Gamma$ a generalised hexagon}

Suppose that $\Gamma$ is a generalised hexagon, with $S_{x} \cong\left[q^{11}\right]: \mathrm{GL}_{2}(q)$. Since $\left|\mathrm{GL}_{2}(q)\right|=$ $q\left(q^{2}-1\right)(q-1)$

$$
|\mathcal{P}|=\left|S: S_{x}\right|=\left(q^{4}-q^{2}+1\right)\left(q^{2}+1\right)^{2}\left(q^{3}+1\right) .
$$

Equivalently (subtracting 1 from both sides),

$$
s^{3} t^{2}+s^{2}(t+1)+s(t+1)=q^{11}+q^{9}+q^{8}+q^{6}+q^{5}+q^{3}+q^{2} .
$$

Now, $S$ acts primitively and distance-transitively with stabiliser $\left[q^{11}\right]: \mathrm{GL}_{2}(q)$ on the points of a generalised octagon of order $\left(q^{2}, q\right)$, namely the point-line dual of the generalised octagon from case (i). The nontrivial subdegrees are [13, Section 4.9.4]

$$
n_{1}:=q^{2}(q+1), \quad n_{2}:=q^{5}(q+1), \quad n_{3}:=q^{8}(q+1), \quad n_{4}:=q^{11} .
$$


For $x \in \mathcal{P}$, we again have (6), and $S_{x}$ must preserve the sets $\Gamma_{i}(x), i \in\{2,4,6\}$, so each $\left|\Gamma_{i}(x)\right|$ is equal to a sum of the subdegrees $n_{1}, \ldots, n_{4}$, as in Section 5.2. We show that this leads to a contradiction.

Claim 1: $\left|\Gamma_{2}(x)\right|=n_{1}$. The proof of the claim is by contradiction. If not, then $\left|\Gamma_{2}(x)\right| \geqslant n_{2}=q^{5}(q+1)$. Since $s, t \geqslant 2$, and so in particular $t \geqslant \frac{2}{3}(t+1)$, it follows that

$$
\begin{aligned}
& \left|\Gamma_{4}(x)\right| \geqslant \frac{2}{3} s^{2}(t+1)^{2}=\frac{2}{3}\left|\Gamma_{2}(x)\right|^{2} \geqslant \frac{2}{3} q^{10}(q+1)^{2}, \\
& \left|\Gamma_{6}(x)\right| \geqslant 2 s^{2} t^{2} \geqslant \frac{4}{3}\left|\Gamma_{4}(x)\right| \geqslant \frac{8}{9} q^{10}(q+1)^{2} .
\end{aligned}
$$

Since the left-hand side of $(7)$ is $\left|\Gamma_{2}(x)\right|+\left|\Gamma_{4}(x)\right|+\left|\Gamma_{6}(x)\right|$, this implies the following inequality, which is false for $q \geqslant 8$ :

$$
\frac{14}{9} q^{10}(q+1)^{2}+q^{5}(q+1) \leqslant q^{11}+q^{9}+q^{8}+q^{6}+q^{5}+q^{3}+q^{2} .
$$

Claim 2: $\left|\Gamma_{4}(x)\right|=n_{2}$. The proof is again by contradiction. If not, then $\left|\Gamma_{4}(x)\right| \geqslant$ $n_{3}=q^{8}(q+1)$, because $\left|\Gamma_{2}(x)\right|=n_{1}=q^{2}(q+1)$ by Claim 1. This implies the following inequality, which is false for $q \geqslant 8$ :

$$
q^{6}=\frac{q^{8}(q+1)}{q^{2}(q+1)} \leqslant \frac{\left|\Gamma_{4}(x)\right|}{\left|\Gamma_{2}(x)\right|}=\frac{s^{2} t(t+1)}{s(t+1)}=s t<s(t+1)=q^{2}(q+1) .
$$
hence

By Claims 1 and 2, we must have $\left|\Gamma_{6}(x)\right|=n_{3}+n_{4}=q^{8}\left(q^{3}+q+1\right)>q^{9}\left(q^{2}+1\right)$, and

$$
s>\frac{s^{3} t^{2}}{s^{2} t(t+1)}=\frac{\left|\Gamma_{6}(x)\right|}{\left|\Gamma_{4}(x)\right|}>\frac{q^{9}\left(q^{2}+1\right)}{q^{5}(q+1)}=\frac{q^{4}\left(q^{2}+1\right)}{q+1} .
$$

This, however, contradicts $s(t+1)=q^{2}\left(q^{2}+1\right)$ (namely Claim 1 ).

\subsection{Cases (iii) $-(\mathrm{ix})$}

We now deal with cases (iii)-(ix), for which we use Lemma 2(i) to contradict the equality $|\mathcal{P}|=\left|S: S_{x}\right|$. First suppose that $S_{x}$ is isomorphic to either $\mathrm{SU}_{3}(q): C_{2}$ or $\operatorname{PGU}_{3}(q): C_{2}$. In either case, we have $\left|S_{x}\right|=2 q^{3}\left(q^{3}+1\right)\left(q^{2}-1\right)$, and hence

$$
|\mathcal{P}|=\frac{1}{2} q^{9}\left(q^{6}+1\right)\left(q^{2}+1\right)(q-1)=2^{9 m-1}\left(2^{6 m}+1\right)\left(2^{2 m}+1\right)\left(2^{m}-1\right)<2^{18 m+1} .
$$

However, Lemma 2(i) with $a=9 m-1$ gives $|\mathcal{P}|>2^{27 m-3}$, which is a contradiction.

If $S_{x} \cong \operatorname{Sz}(q)$ $C_{2}$, then $\left|S_{x}\right|=2 q^{4}\left(q^{2}+1\right)^{2}(q-1)^{2}$, so

$|\mathcal{P}|=\frac{1}{2} q^{8}\left(q^{4}-q^{2}+1\right)\left(q^{3}+1\right)(q+1)=2^{8 m-1}\left(2^{4 m}-2^{2 m}+1\right)\left(2^{3 m}+1\right)\left(2^{m}+1\right)<2^{16 m+1}$,

contradicting Lemma 2(i) with $a=8 m-1$, which gives $|\mathcal{P}|>2^{24 m-3}$.

If $S_{x} \cong \operatorname{Sp}_{4}(q): C_{2}$, then $\left|S_{x}\right|=2 q^{4}\left(q^{4}-1\right)\left(q^{2}-1\right)$, so

$$
|\mathcal{P}|=\frac{1}{2} q^{8}\left(q^{6}+1\right)\left(q^{2}-q+1\right)=2^{8 m-1}\left(2^{6 m}+1\right)\left(2^{2 m}-2^{m}+1\right)<2^{16 m},
$$

contradicting Lemma 2(i) with $a=8 m-1$, which gives $|\mathcal{P}|>2^{24 m-3}$. 
Now suppose that $S_{x} \cong{ }^{2} F_{4}\left(q_{0}\right)$, where $q=q_{0}^{r}$ with $r$ prime. Writing $q_{0}=2^{\ell}$, we have

$$
|\mathcal{P}|=2^{12 \ell(r-1)} \frac{\left(2^{6 r \ell}+1\right)\left(2^{4 r \ell}-1\right)\left(2^{3 r \ell}+1\right)\left(2^{r \ell}-1\right)}{\left(2^{6 \ell}+1\right)\left(2^{4 \ell}-1\right)\left(2^{3 \ell}+1\right)\left(2^{\ell}-1\right)}<2^{26 \ell(r-1)+4}
$$

However, Lemma 2(i) with $a=12 \ell(r-1)$ gives $|\mathcal{P}|>2^{36 \ell(r-1)}$, a contradiction (because $\ell \geqslant 1)$.

Finally, suppose that $S_{x}$ is as in one of the cases (viii)-(x). Then the highest power of 2 dividing $\left|S_{x}\right|$ is $2^{5}$ (arising in case (ix)), so $|\mathcal{P}|=\left|S: S_{x}\right|$ is divisible by $2^{12 m-5}$, and Lemma 2(i) therefore gives $|\mathcal{P}|>2^{36 m-15}$. On the other hand, we certainly have $|\mathcal{P}|<|S|<2^{30 m}$, which is a contradiction (because $36 m-15 \leqslant 30 m$ if and only if $m \leqslant 5 / 2$, but we have $m \geqslant 3$ ).

\section{Acknowledgements}

The authors thank John Bamberg for helpful discussions, and in particular for suggesting the arguments for cases (i) and (ii) in Section 5.

\section{References}

[1] J. Bamberg, S. P. Glasby, T. Popiel, C. E. Praeger and C. Schneider, "Point-primitive generalised hexagons and octagons", preprint, 2014, arXiv:1410.3423.

[2] J. N. Bray, D. F. Holt and C. M. Roney-Dougal, The Maximal Subgroups of the Low-Dimensional Finite Classical Groups, London Math. Soc. Lecture Note Ser. 407, Cambridge University Press, Cambridge, 2013.

[3] F. Buekenhout and H. van Maldeghem, "Remarks on finite generalized hexagons and octagons with point-transitive automorphism groups", in: F. De Clerck (Ed.), Finite geometry and combinatorics, London Math. Soc. Lecture Note Ser. 191, Cambridge University Press, Cambridge, 1993, pp. 89-102.

[4] F. Buekenhout and H. Van Maldeghem, "Finite distance-transitive generalized polygons", Geom. Dedicata 52 (1994) 41-51.

[5] J. D. Dixon and B. Mortimer, Permutation Groups. Springer-Verlag, New York, 1996.

[6] W. Feit and G. Higman, "The nonexistence of certain generalized polygons", $J$. Algebra 1 (1964) 114-131.

[7] S. Guest and C. E. Praeger, "Proportions of elements with given 2-part order in finite classical groups of odd characteristic", J. Algebra 372 (2012) 637-660.

[8] P. B. Kleidman, "The maximal subgroups of the Chevalley groups $G_{2}(q)$ with $q$ odd, the Ree groups ${ }^{2} G_{2}(q)$, and their automorphism groups", J. Algebra 117 (1988) $30-71$.

[9] G. Malle, "The maximal subgroups of ${ }^{2} F_{4}\left(q^{2}\right)$ ", J. Algebra 139 (1991) 52-69. 
[10] C. Schneider and H. Van Maldeghem, "Primitive flag-transitive generalized hexagons and octagons", J. Combin. Theory Ser. A 115 (2008) 1436-1455.

[11] H. Van Maldeghem, Generalized polygons, Birkhäuser/Springer Basel AG, Basel, 1998.

[12] M. Suzuki, "A new type of simple groups of finite order", Proc. Nat. Acad. Sci. U.S.A. 46 (1960) 868-870.

[13] R. A. Wilson, The finite simple groups, Graduate Texts in Mathematics 251, Springer-Verlag, London, 2009. 